positive syphilis test $[\mathrm{RR}=1.59 ; 95 \% \mathrm{CI}=1.33-1.89]$. Postpartum, $39(16 \%)$ intervention and 21 (9\%) control men reported known HIV-positive status during pregnancy. Despite increased knowledge of HIV-positive status within the intervention group, men were less likely to link to HIV care services $[\mathrm{RR}=0.69 ; 95 \% \mathrm{CI}=0.50-0.96]$, as $41 \%$ (16 of 39$)$ of men were newly diagnosed with HIV. No differences were observed for uptake of male circumcision within the study period $[\mathrm{RR}=1.59 ; 95 \% \mathrm{CI}=0.89-2.87]$ or attendance of subsequent clinic-based antenatal care with the female partner $[\mathrm{RR}=1.11 ; 95 \% \mathrm{CI}=0.85-1.41]$.

Conclusion Home-based couple education and testing resulted in greater uptake of clinic-based STI consultation services among men. However, increased knowledge of HIV-status in the home did not lead to increased HIV care service uptake for men, potentially because there was a greater proportion of men with new HIV diagnoses in the intervention arm. This group of newly diagnosed men should be targeted with research to increase linkage and engagement to HIV care.

\section{P2.21 FEASIBILITY STUDY FOR THE DEVELOPMENT OF A RAPID LATERAL FLOW POINT OF CARE TEST FOR CONGENITAL SYPHILIS}

${ }^{1} J o h n$ Deutsch, 'Yetunda F Fakile, ${ }^{1}$ Ellen Kersh, ${ }^{2} J a v a n$ Esfandiari, ${ }^{3}$ Ardianne Cruz. ${ }^{1}$ The Centre for Disease Control and Prevention, Atlanta, GA, USA; ${ }^{2}$ Chembio Diagnostic Systems, Medford, NY, USA; ${ }^{3}$ UCONN Health Centre, Farmington, CT, USA

10.1136/sextrans-2017-053264.197

Introduction The World Health Organisation (WHO) estimates there are 713600 to $1,575,000$ cases of congenital syphilis worldwide annually. Congenital syphilis can lead to several health conditions at birth. Currently there is no rapid point of care test available for its diagnosis.Here we introduce a novel rapid point of care test for congenital syphilis. The objective of this test includes the ability to test whole blood or serum taken from the infant or cord blood while blocking the mother's IgG antibodies.

Methods Thirty-four (34) syphilis positive and negative gestational serum samples and five (5) positive congenital syphilis samples, were obtained from University of Connecticut Health Centre. Twenty-two (22) samples were RPR reactive. Chembio DPP Syphilis IgG and IgM test devices were obtained from the manufacturer. The required number of $\operatorname{IgG}$ and IgM devices were modified at the CDC to include an IgG blocker in the device to produce four different assay devices used for this study. Each serum sample was tested in the four separate devices. Results were reported based on the patterns observed in the assay windows.

Results All (17/17) the RPR positive gestational samples were positive $(100 \%)$, and 5 of 5 congenital positive samples were positive (100\%) using the IgM device with IgG blocking agent. Only $5 / 17$ of gestational samples $(29.4 \%)$ and $1 / 5$ congenital samples (20\%) were positive using the IgM device without $\operatorname{IgG}$ blocking agent. For the $\operatorname{IgG}$ device with the $\operatorname{IgG}$ blocking agent all samples were negative. The IgG device without the blocking reagent resulted in the detection of 19/ $22(86.4 \%)$ of the RPR positive samples. With the exception of one sample all negative samples were negative by all 4 test devices.

Conclusion Only a limited number of positive and negative gestational and congenital syphilis samples were evaluated in this study, due to the short supply of such rare samples. It is apparent from this limited data that by blocking the mother's IgG an increase in sensitivity can be achieved. More in depth studies are necessary in order to prove the concept.

\section{P2.22 SURVEILLANCE OF HIV-1 TRANSMITTED DRUG RESISTANCE AMONG DRUG-NAÏVE POPULATIONS IN RIO DE JANEIRO, BRAZIL}

${ }^{1}$ José Carlos Couto Fernandez, ${ }^{2}$ Giovanni Ravasi, ${ }^{3}$ Michelle Das Neves, ${ }^{3}$ Jose Henrique Pilotto, ${ }^{3}$ Carlos Silva de Jesus, ${ }^{3}$ Mariza Gonçalves Morgado. 'Laboratory of AIDS and Molecular Immunology, Oswaldo Cruz Foundation-loc/Fiocruz, Rio de Janeiro - RJ, Brazil; ${ }^{2}$ Pan-American Health Organization-PAHO, World Health Organisation, Washington DC, USA; ${ }^{3}$ Oswaldo Cruz Foundation-loc/Fiocruz, Rio de Janeiro - RJ, Brazil

\subsection{6/sextrans-2017-053264.198}

Introduction The WHO Global HIV drug resistance network (HIV-ResNet), was stablished to monitor the emergence and help to control the transmission of HIV-1 drug resistant strains. The TDR is progressively increasing over the last years in some Brazilian regions, mainly where the epidemic is concentrated. This study evaluated the trends in the prevalence of TDR mutations and dynamic of subtypes, among drug-naïve HIV-1 infected individuals from vulnerable group populations in the context of the WHO HIV-ResNet.

Methods We analysed a total of 536 HIV-1 sequences collected during 2005 to 2014, targeting drug naïve pregnant woman from four public antenatal care units and 159 recently diagnosed $(<1$ year) individuals identified in VCTs in all Rio de Janeiro state. The profiles of TDR mutations were evaluated using the updated WHO transmitted resistance mutation list and HIV-1 genetic diversity evaluated by phylogenetic analysis.

Results Overall, the prevalence of TDR was 12.5\% (CI95\%, $7.15 \%$ to $16.5 \%)$ being, $5.8 \%(\mathrm{CI} 95 \%, 2.5 \%$ to $9.15 \%)$ to the nucleoside reverse transcriptase inhibitors (NRTIs), 3\% (CI95\%, $0.1 \%$ to $4.85 \%)$ to non-nucleoside inhibitors (NNRTIs) and $3.7 \%$ (CI95\%, $1.24 \%$ to $7.5 \%)$ to protease inhibitors (PIs). Both studied groups showed similar TDR prevalence for all drug classes. The timidine-associated mutations (TAMs) and $\mathrm{M} 184 \mathrm{~V}$ were the most prevalent TDR mutations found in RT gene, followed by K103N, T215 revertants and F77L. The M46I PI associated mutation was the more frequent, followed by V82A and L90M. HIV-1 subtype B was the most prevalent (80\%), followed by F1 (7.5\%), subtype $\mathrm{C}(4 \%)$ and $\mathrm{BF}$ recombinants (3.5\%). In addition to non-B HIV-1 subtype A1, G, CRF02_AG, CRF31_BC, FC and DF recombinants, identified in $4 \%$ of genotyped samples. Significant difference was observed in the two groups, where subtype $F(12 \%)$ was more prevalent in pregnant woman, while subtype $\mathrm{C}$ prevalent in new diagnosed subjects (5.9\%). Conclusion This work tried to study trends of HIV-1 TDR and the genetic diversity in Rio de Janeiro state, the second major HIV/AIDS epidemic in Brazil. The results demonstrated 
a sustained low prevalence of TDR to the PIs, recent accumulation of resistance associated to the NRTIs and reduction to NNRTIs over the years. The time trend of TDR observed, seem to reflect changes in antiretroviral therapy in Brazil over time. HIV-1 subtype B was the most prevalent in the study, but the increasing prevalence of subtype $\mathrm{C}$ and the identification of others non-B and recombinants infections, suggest the recent introduction and spreading of these viruses, respectively south Brazil and African countries in Rio de Janeiro.

Support: Oswaldo Cruz Foundation-IOC/FIOCRUZ, Brazilian Ministry of Health (DDAHV-CQV/MS), Pan-American Health Organization-PAHO and World Health Organization-WHO

\section{P2.23 HEPATOTOXICITY AND ANAEMIA CO-MORBIDITY IN TREATED AIDS PATIENTS IN FUNDONG SUB DIVISION IN THE NORTHWEST REGION OF CAMEROON}

${ }^{1}$ Lem Edith Abongwa, ${ }^{2}$ Anthony Kebira Nyamache, ${ }^{1}$ Nantia Akono, ${ }^{1}$ Stanley Sunjo, ${ }^{1}$ Vera Nange, ${ }^{1}$ Hebert Balan, ${ }^{3}$ Fokunang Charles, ${ }^{2}$ Paul Okemo, ${ }^{3}$ Judith Torimiro. ${ }^{1}$ University of Bamenda, Faculty of Science, Department of Biological Sciences, P.O. Box 39, Bambili, Bamenda - Cameroon; ${ }^{2}$ School of Pure and Applied Sciences, Department of Microbiology, Kenyatta University, P.O. Box 43, Nairobi, Kenya; ${ }^{3}$ Faculty of Medicine and Biomedical Sciences, University of Yaounde I, Yaounde, Yaounde - Cameroon

\subsection{6/sextrans-2017-053264.199}

Introduction Hepatotoxicity and anaemia are relevant adverse effects of ART and can cause interruption of therapy and death. However, there is dearth of information on hepatotoxicity and anaemia co-morbidity especially in rural areas. The aim of the study was to identify the prevalence of Hepatotoxicity and Anaemia co-morbidity among HIV treated patients.

Methods A total of 150 drug naïve patients visiting the day hospital in Fundong District Hospital were recruited into the study from January-March 2015 and follow up for 18 months. Baseline and 18 months levels of CD4 counts, alanine transaminase(ALT), and aspartate transaminase(AST) and Haemoglobin concentration $(\mathrm{Hb})$ were determined. HIV was diagnosed using Alere determine HIV rapid test kit and Bioline or Oral Quick test kit for the confirmatory test. CD4 counts were determined using the Alere Pima CD4 cartridge machine. $\mathrm{Hb}$, ALT and AST counts were determined by colometric enzymatic reaction using the urit 3300 machine and classified based on age and sex.

Results The majority of patients were female $115(76.7 \%)$ and belonged to the $<30$ years age range 48 (32\%). The prevalence of anaemia decreased from $86(57.3 \%)$ to $69(45.6 \%)$ at the end of the study period. In all 46 (30.7\%) patients had hepatotoxicity and anaemia co-morbidity which was higher in the age group $<30$ years $30(41.7 \%)$ and in female 37 $(32.2 \%)$. A total of $1(0.7 \%)$ and $10(6.7 \%)$ patients develop severe hepatotoxicity using ALT and AST respectively. The prevalence of hepatotoxicity was higher in male $31.4 \%$ and $62.9 \%)$ and in the age group 30-39 years $(29.5 \%$ and $68.2 \%)$ for ALT and AST, respectively. The prevalence of anaemia and elevated AST and ALT were higher in persons with CD4 <200 cells $/ \mu$ l. There was a significant correlation between $\mathrm{CD} 4$ and $\mathrm{Hb}(\mathrm{r}=0.193), \mathrm{CD} 4$ and $\operatorname{ALT}(\mathrm{r}=-0.149)$ and CD4 and AST(r=-0.193).

Conclusion Hepatotoxicity especially Grades 1-2 and not anaemia is a significant adverse effect of ART upon time.

\section{$\mathrm{P} 2.24$}

AWARENESS AND INTEREST IN PRE-EXPOSURE PROPHYLAXIS (PREP) AMONG PATIENTS RECEIVING SERVICES AT A PUBLIC SEXUALLY TRANSMITTED DISEASES (STD) CLINIC IN A HIGH PREVALENCE URBAN SETTING

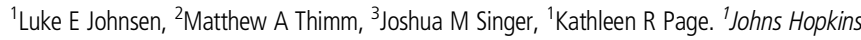
University School of Medicine, Baltimore City Health Department, Baltimore, Md, USA; ${ }^{2}$ Johns Hopkins University School of Medicine, Baltimore, Md, USA; ${ }^{3}$ University of Maryland, College Park, MD, USA

\subsection{6/sextrans-2017-053264.200}

Introduction HIV rates in Baltimore City are among the highest in the United States, with the majority of new infections attributed to male-to-male sexual contact. In 2016, the Baltimore City Health Department (BCHD) implemented a PreExposure Prophylaxis (PrEP) program at their sexually transmitted diseases (STD) clinics. We assessed awareness, interest, and sources of knowledge about PrEP among patients served at these clinics.

Methods We surveyed a convenience sample of 1675 patients who attended BCHD clinics between 4/12/2016 and 10/3/ 2016. Participants were provided a self-administered survey that assessed awareness, interest, and sources of knowledge about PrEP, as well as age, race, sex, and sexual preference. Univariate and multivariable logistic regression analysis examined predictors of awareness and interest. Data collection will continue in 2017 to determine if awareness and interest change over time.

Results Mean age of participants was $32.1 \quad(\mathrm{SD}=11.8)$ and $61.0 \%$ were male. $91.1 \%$ of participants self-identified as African American, 5.7\% as white, and 3.2\% as "Other." $20.0 \%$ of the participants were aware of PrEP and $42.2 \%$ were interested in PrEP. Less than 1\% of all participants and $9.2 \%$ of men who have sex with men (MSM) reported current PrEP use. White participants were more likely to be aware of PrEP than African Americans $(O R=1.76, p=0.026)$, but there was no significant difference in interest between these groups $(p=0.122)$. By univariate analysis men were more likely to be aware of PrEP than women $(\mathrm{OR}=1.38, \mathrm{p}=0.013)$, but multivariable analysis eliminated this significant difference $(p=0.081)$. MSM were significantly more likely to be aware $(\mathrm{OR}=14.29, \mathrm{p}<0.001)$ and interested $(\mathrm{OR}=1.59, \mathrm{p}=0.024)$ in PrEP. Sources of knowledge included healthcare providers (40.0\%), friends (24.7\%), and television (16.4\%).

Conclusion MSM receiving care at the Baltimore City STD Clinics are significantly more likely to be aware and interested in PrEP, but few are taking PrEP, highlighting a need to improve access and promote uptake in this high risk population.

\section{P2.25 PULMONARY RHODOCOCCOSIS WITH BACTEREMIA AS AN AIDS DEFINING INFECTION: CASE REPORT}

Marcos Davi Gomes de Sousa, Juliana Neves Ferreira de Oliveira, Ofelio Alberto Manuel Andrea Varon, Maria Cristina da Silva Lourenço, Erica Aparecida dos Santos, Cristiane Lamas. National Institute of Infectology Evandro Chagas, Rio de Janeiro - RJ, Brazil

\subsection{6/sextrans-2017-053264.201}

Introduction In endemic countries for tuberculosis (TB), HIVTB coinfection is very common. However, other bacteria such as Rhodococcus produce similar clinical and radiological 